\title{
GLORIA EVANGELINA ANZALDÚA: REPRESENTAÇÃO E INTELECTUALIDADE EM BORDERLANDS/LA FRONTERA
}

\author{
Carlos Vinícius da Silva Figueiredo* \\ Vera Lúcia Harabagi Hanna**
}

\begin{abstract}
RESUMO: O contexto histórico-cultural e literário de grande produtividade nos Estados Unidos tem fomentado intensivamente as literaturas imigrantes e de identidades em trânsito, proporcionando a criação de uma vasta obra representante do solo cultural da fronteira entre México e Estados Unidos. Uma representante deste espaço fronteiriço é Gloria Evangelina Anzaldúa, que transitou da poesia à prosa, a exemplo do livro Borderlands/La Frontera: the new mestiza (1987), revelando o surgimento de um rótulo em particular, como o de literatura chicana. O objetivo principal deste artigo é analisar a figura da escritora Gloria Anzalúa enquanto intelectual que ultrapassou os limites da fronteira para se estabelecer como questionadora do status quo. Percebe-se que tanto as palavras quanto as ações de Anzaldúa materializam uma leitura de significativa representação e resistência ao poder hegemônico estabelecido, pois, possibilita o (re) pensar das próprias fronteiras de seus leitores. Para tanto, a metodologia que subsidia este texto volta-se para as reflexões teórico-críticas acerca dos estudos pós-colonialistas, sobretudo as obras, Mignolo (2007) e Hawley (2001) e ainda obras de exegese e fortuna crítica sobre Anzaldúa, como, Keating (2009), Cantú (2012) e Malvezzi (2010). Desta forma, a vida e obra de Gloria Anzaldúa constituem-se, em um legado da história daqueles que estão na fronteira e vivem a difícil realidade deste entre-lugar.
\end{abstract}

ABSTRACT: The highly productive historical-cultural and literary context in the United States has intensively fostered immigrant and identity literature in transit, providing the creation of a vast work representing the cultural land of the border between Mexico and the United States. A representative of this border area is Gloria Evangelina Anzaldú, who moved from poetry to prose, like the book Borderlands / La Frontera: the new mestiza (1987), revealing the emergence of a particular label, such as the Chicana literature. The main objective of this paper is to analyze the figure of the writer Gloria Anzalúa as an intellectual who crossed the boundaries of the border to establish herself as a questioner of the status quo. It is noticed that both Anzaldua's words and actions represents a reading of significant resistance to the established hegemonic power, as it allows the (re) thinking of its readers' own borders. In this way, the methodology that guides this text focuses on the theoretical-critical reflections on post-colonial studies, especially the works, Mignolo (2007) and Hawley (2001), as well as works about Anzaldua, as Keating (2009), Cantú (2012) and Malvezzi (2010). Thus, the life and work of Gloria Anzaldúa are a legacy of the history of those who are on the border and who can perceive the difficult reality of the in-between spaces.

PALAVRAS-CHAVE: intelectual; Anzaldúa; representação.

KEYWORDS: intellectual; Anzaldúa; representation. 
[...] o olhar pós-moderno pôs o intelectual à margem do saber instituído obrigando-o, por conseguinte, a rediscutir conceitos canônicos como o de cultura e de literatura, por exemplo. Daí ser escusado dizer ainda que esse mesmo intelectual não pode mais se valer de conceitos nem de um olhar excludentes diante da sociedade. (NOLASCO, 2007, p.76)

As palavras de Edgar Nolasco (2007) materializam o pensamento de que não há mais espaço para discussões que deixem de lado a sociedade. Há de se pensar, na contemporaneidade, nas diferentes formas de olhar para o outro como sujeito dotado de cultura, independentemente de seu status social ou localidade. Olhar este, que marcou o projeto intelectual e literário de Gloria Evangelina Anzaldúa, dialogando como os sujeitos que ocupam o espaço fronteiriço dos EUA e o México.

Entende-se, neste artigo, que ocupar o lugar de questionadora do status quo e viver uma vida voltada a ouvir o próximo são características presentes na biografia de Anzaldúa. Soma-se a isso, a capacidade da autora de transitar entre os mundos da academia e sua vivência na fronteira, sem esquecer sua herança cultural indígena e chicana.

Gloria Evangelina Anzaldúa (1942-2004), filha mais velha da sexta geração de mexicanos de Rio Grande Valley, no Sul do Texas, foi uma teórica feminista chicana, cujo trabalho transcendeu as fronteiras de seu locus e deu voz às mulheres de $\operatorname{cor}^{1}$, num contexto de discurso hegemônico. Autora de títulos como Bordelands/La Frontera (1987), This bridge called my back (1983), This Bridge Called My Back: Writings by Radical Women of Color (1981), Making Face, Making Soul/Haciendo Caras: Creative and Critical Perspectives by Feminists-of-Color (1990), Prietita and the Ghost Woman/Prietita y la Llorona (1995), nos quais, evidenciam-se o diálogo sobre a identidade cultural, identidade chicana, feminismo e fronteira.

Destaca-se, nesse sentido, o projeto intelectual de Anzaldúa enquanto chicana, homossexual, poetiza e nepantlera, preocupada em materializar em suas palavras "a vida na escuridão, a vida nas fronteiras". Observa-se, nesse sentido, o fato de Anzaldúa não escrever sobre as margens, mas, sim, ter ocupado esse lugar e vivido o mundo dos marginalizados, expondo não apenas as fronteiras geográficas que dividem os países, mas também as fronteiras sociais, de gênero e identidade, esquecidas pelo poder hegemônico.

Said (2005) dialoga com este pensamento à medida que aponta o papel público do intelectual como um outsider, um "amador", um perturbador do status quo, afirmando,

${ }^{1}$ Utiliza-se esta tradução do original em inglês "women of colors." Essa definição é recorrente nos textos analisados, tanto os produzidos por Anzaldúa quanto os textos teóricos. 
ainda, que uma das tarefas do intelectual é derrubar os estereótipos e as categorias redutoras que tanto limitam o pensamento humano e a comunicação. Em outras palavras, o principal dever do intelectual é a busca de uma relativa independência em face das pressões do mundo contemporâneo.

Dessa forma, entende-se que o intelectual é aquele que abre espaço através da reflexão, para que o outro tenha lugar, para que o que foi silenciado venha a ser falado. Daí talvez iniciar-se um diálogo com o que Anzaldúa propôs em sua produção e sua vida, ao se definir como uma nepantlera ${ }^{2}$, procurando transitar entre os diferentes mundos e descolonizar o discurso e poder hegemônico. Sendo oportuno rememorar aqui as palavras de Gramsci ao afirmar que "todos os homens são intelectuais, mas nem todos os homens desempenham na sociedade a função de intelectuais." (p.7) Nesse sentido, evidencia-se a vertente crítica descolonialista como perspectiva interessante para este debate.

Segundo Gros-foguel (2007), ao discutir sobre a relação entre colonialidade e poder, aponta que o mundo ainda não foi completamente descolonizado. Segundo o autor, a primeira descolonização (iniciada no século XIX pelas colônias espanholas e em seguida, no século XX pelas colônias inglesas e francesas) foi incompleta, uma vez que se limitou a independência jurídico-política das periferias. Em contrapartida, a segunda descolonização, dirige-se a heterarquia das múltiplas relações raciais, étnicas, sexuais, epistêmicas, econômicas e de gênero das quais a primeira descolonização deixou intactas. Completa o pesquisador, afirmando que: "[...] el mundo de comienzos del siglo XXI necesita una decolonialidad ${ }^{3}$ que complemente la descolonización llevada a cabo en los siglos XIX y XX. Al contrario de esa descolonización, la decolonialidad es un processo de resignificación a largo plazo, que no puede reducir a un acontecimiento jurídicopolítico". (GROS-FOGUEL, 2005, p.18)

Com isso, a descolonialização ${ }^{5}$ emerge como alternativa de se refletir sobre o processo de dominação vivenciado na modernidade. Para Mignolo:

\footnotetext{
${ }^{2}$ Nepantla é uma palavra de origem Nahuat que significa em situação de "entre-lugar", contexto no qual os Astecas se viram, no século XVI, entre a sabedoria dos anciãos e a contínua colonização espanhola. Nepantlera é um adjetivo utilizado para caracterizar Anzaldúa como uma facilitadora, uma pessoa capaz de auxiliar outras a atravessar os entre-lugares/ entre-mundos.

${ }^{3}$ Observa-se que a tese utilizará o termo descolonial para traduzir o termo decolonial (inglês e espanhol).

${ }^{4}$ Tradução livre: [...] o mundo do início do século XXI precisa de um descolonialidade que complementa a descolonização realizada nos séculos XIX e XX. Ao contrário do que dessa descolonização, a descolonialidade é um Processo de ressignificação de longo prazo, que não pode ser reduzido a um acontecimento jurídico-político.

${ }^{5}$ Será utilizado neste trabalho o termo descolonização para se referir a tradução de decolonialidad em espanhol.
} 
La decolonialidad es, entonces, la energía que no se deja manejar por la lógica de la colonialidad, ni se cree los cuentos de hadas de la retórica de la modernidad. Si la decolonialidad tiene una variada gama de manifestaciones [...], el pensamiento decolonial es, entonces, el pensamiento que se desprende y se abre [...], encubierto por la racionalidad moderna, montado y encerrado en las categorías del griego y del latín y de las seis lenguas imperiales europeas modernas. (MIGNOLO apud CASTRO-GÓMEZ Y GROSFOGUEL, 2007, p. $27)^{6}$

Corrobora com essa discussão, a afirmação de John C. Hawley, em sua Encyclopydia of Postcolonial Studies (2001), ao apontar que Anzaldúa foi "uma ativista política, pois sua escrita se constituía em um movimento de encorajamento e transformação social". Ainda segundo Hawley, ao editar antologias, Anzaldúa contribuiu para uma "consciência social, construiu comunhão e proporcionou um espaço de diálogo crucial para seu tempo". Segundo o autor:

Anzaldúa provokes "others" to "decolonize" themselves. She deconstructs categories of fixed identities to encourage bridges across difference. She defines creative acts as forms of political activism and identifies the advantages of taking part in the culture by helping to build it, making space for the self and for others in an ongoing process. ${ }^{7}$ (HAWLEY, 2001, p. 28).

Considerando a afirmação de Hawley (2001) de que Anzaldúa provoca as pessoas a se descolonizarem, o autor mantém contato direto com este artigo, quando trata da necessidade de discutir sobre os seres subalternos sob uma nova perspectiva. Uma perspectiva que possibilite o diálogo e uma representação que parta, inicialmente, pelo ouvir a história do outro, assim como Anzaldúa pôde contar a sua história. Ao se referir "others", como outros, Hawley abre espaço para se pensar naqueles que não estão representados pelo status quo, para àqueles que estão nas margens e nas sombras, impelindo-os a se desconstruírem para alcançarem seus objetivos.

Continua Hawley (2001), mencionando que Anzaldúa desafia o discurso imperialista e que “[...] Her articulation of the 'borderlands' (psychological, sexual, geographic, spiritual, linguistic, and theoretical) posits a border-crossing trope useful for theories of

\footnotetext{
6 Tradução livre: A descolonialidade, então, é a energia que não se deixa ser operada pela lógica da colonialidade, nem cre em contos de fadas da retórica da modernidade. Se a descolonialidade tem uma vasta gama de manifestações [...], o pensamento descolonial, então, é o pensamento que emerge e se abre [...], escondido pela racionalidade moderna, montado e travado nas categorias de grego e do latin e as seis línguas imperiais européias modernas.

7 Tradução livre: Anzaldúa provoca "outros" para "descolonizar" a si mesmos. Ela desconstrói categorias de identidades fixas para encorajar pontes através da diferença. Ela define os atos criativos como formas de ativismo político e identifica as vantagens de participar da cultura, ajudando a construí-la, fazendo o espaço para si e para os outros em um processo contínuo.
} 
subjectivity."8(HAWLEY, 2001, p. 28) O autor assevera, durante seu texto, que Anzaldúa ao demonstrar a interpenetração de tantas fronteiras, a exemplo da ética, gênero, linguística, sexual e religiosa, expõe as forças políticas que constroem as fronteiras para dividir e conquistar àqueles menos empoderados.

Para Hawley (2001), o fato é que as fronteiras estão em estado de constante transição, o que, por sua vez, dá ímpeto aos movimentos de mudança social e identifica o processo contínuo de subjetividades múltiplas em um indivíduo. Nesse sentido, a obra de Anzaldúa contesta a noção filosófica ocidental de estabilidade, de sujeito unificado, e do discurso e voz autoritários assumidos pelo homem branco, que sempre separa os "outros" nas relações de poder.

Os "outros", mencionados por Hawley, podem ser encontrados em todos os lugares, contudo, não são vistos ou ouvidos. Para Anzaldúa esses seres subalternos são os "transgresors", "alien", "strangers", "inhuman", "subhuman", adjetivos utilizados no livro Borderlands/La Frontera (2012). Trata-se de seres que desafiam o mundo por tentarem viver nele, e questionam toda a noção de estabilidade do mundo ocidental, que por sua vez, não permite que esses seres ocupem seus espaços, resistindo a todos os rótulos a eles atribuídos pela sociedade dominante. Desta forma, concorda-se com Hawley ao posicionar a ideologia de Anzaldúa como incapaz de negar qualquer parte de sua identidade, das culturas que a compõem, pois tal ação representa um modelo que começa a ser referenciado na academia e consegue refletir sobre as realidades do mundo pós-moderno.

Na esteira de Hawley (2001), depreende-se que o texto de Anzaldúa constitui-se em um convite ao reconhecimento e apreciação do "outro", do diferente. Configura-se na possibilidade de se refletir sobre o mundo a partir de experiências pessoais, e daquilo que é acreditado, pois, a autora encoraja seu leitor a pensar sobre um projeto emancipatório. Tal projeto, já identificado nos estudos pós-coloniais, encoraja os leitores a se questionar sobre quem tem o poder ou quem tem voz. Tal ato materializa o cerne do projeto intelectual de Anzaldúa: o de resistência cultural. Para tanto, Hawley (2001) assevera que "[...] Postcolonial people must live within this tension between the clockwise of the colonizing cultures and the counterclockwise of the colonized cultures."(HAWLEY, 2001, p. 29).

Ainda cotejando o texto instigante de Hawley (2001), o pesquisador afirma que:

\footnotetext{
8 Tradução livre: desafia os discursos normativos e imperialistas. Sua articulação das "fronteiras" (psicológica, sexual, geográfica, espiritual, lingüística e teórica) postula um tropo de fronteira útil para as teorias da subjetividade.
} 
Seeing identity as an ongoing process, Anzaldúa embodies a postcolonial strategic resistance to cultural oppression at the interstices of difference embodied by ethinic minorities, women, lesbians, the poor, and the "othered" groups. Anzaldúa reveals the inextricability of self and actively producing self in a social context; she claims that by changing ourselves, we change the world. ${ }^{9}$ (HAWLEY, 2001, p. 28)

Observa-se, nas palavras de Hawley, que Anzaldúa consegue realizar um movimento simultâneo entre o "eu”, em seu grau mais íntimo, e sua respectiva expansão para o mundo, portanto, Anzaldúa, em sua obra, consegue ao mesmo tempo discutir a (re)construção da sociedade à (re)criação do "eu", conectando o eu e a sociedade, a exemplo do " $P$ " e o "we" presentes em grande parte da obra. Segundo a própria autora, "To write, or to be a writer, I have to trust and believe in myself as the speaker, as a voice for the images. I have to believe that I can communicate with images and words and that I can do it well" (ANZALDÚA, 2012, p. 95) ${ }^{10}$.

É oportuno lembrar que Anzaldúa também realiza esse movimento de conexão entre as culturas, quando utiliza do "Spanglish". Tal ação, em sua escrita, oportuniza o acesso a diversos grupos, rompendo, assim, o discurso teórico acadêmico por meio da troca de códigos que amplia seu público de leitores e projeta ainda mais sua resistência poderosa contra os padrões estabelecidos. Como pode ser observado na seguinte passagem:

The Gringo, locked into the fictions of White superiority, seized complete political power, stripping Indians and Mexicans of their land while their feet were still rooted in it. Con el destierro y el exilio fuimos desuñados, destroncados, destripados-we were jerked out by the roots, truncated, disemboweled, dispossessed, and separated from our identity and our history. (ANZALDÚA, 2012, p.29-30) ${ }^{11}$

\footnotetext{
9 Tradução livre: Ao ver a identidade como um processo contínuo, Anzaldúa encarna uma resistência estratégica pós-colonial à opressão cultural nos interstícios da diferença incorporada pelas minorias étnicas, as mulheres, as lésbicas, os pobres e os grupos "alheios". Anzaldúa revela a inextricabilidade do "eu" e a produção ativa do "eu" em um contexto social; Ela assevera que mudando a nós mesmos, mudamos o mundo.

${ }^{10}$ Tradução livre: Escrever, ou ser escritora, eu tenho que confiar e acreditar em mim como falante, como uma voz para as imagens. Eu tenho que acreditar que eu posso me comunicar com as imagens e palavras e que eu posso fazer isso bem.

${ }^{11}$ Tradução livre: O Gringo, bloqueado nas ficções de sua superioridade branca, tomou o poder político completo, tirando índios e mexicanos de suas terras, enquanto os seus pés ainda estavam enraizados nela.
} 
Nesse sentido, o movimento de descolonização ganha força, pois possibilita romper os limites das fronteiras estabelecidas que, por sua vez, estão em constante transição e tensão cultural. Esses fatos podem ser lidos na obra de Anzaldúa como movimentos de transformação social, a exemplo de capítulos como "How to tame a wild tongue" e "The New mestiza consciouness", no livro Borderlands/La Frontera, nos quais se expõe as diferentes fronteiras que se fazem presentes no dia a dia da comunidade fronteiriça.

Cabe aqui abrir espaço para refletir como Anzaldúa representa essa metáfora de descolonização na fronteira, uma vez que a autora chicana expõe a ferida colonial que tanto humilha e separa os povos. Segundo Anzaldúa: "The U.S.-Mexican border es una herida aberta where the Third World grates against the first and bleeds. And before a scab forms it hemorrhages again, the lifeblood of two worlds merging to form a third country-a border culture." (ANZALDÚA, 2012, p. 25) ${ }^{12}$ Diante disso, o texto de Anzaldúa apresenta a realidade que permeia a vida fronteiriça de forma contundente. A criação dessa cultura de fronteira representa a luta dos seres subalternos em terem sua identidade e respeito, constantemente excluída pelo poder cultural dos Estados Unidos. Ao grafar com letras maiúsculas "Third World" Anzaldúa marca seu lugar enquanto ser que se encontra fora do poder hegemônico. Esse mesmo terceiro mundo é relacionado com a cultura de fronteira, criada a partir da mistura/hibridização dos dois países México e Estados Unidos.

Desse ponto de vista, ao tratar do quanto o livro Borderlands representa sua vida, Anzaldúa afirma que:

This book, then, speaks of my existence. My preoccupations with the inner life of the Self, and with the struggle of that Self amidst adversity and violation; with the confluence of primordial images; with the unique positionings consciousness takes at these confluent streams; and with my almost instinctive urge to communicate, to speak, to write about life on the borders, life in the shadows. (ANZALDÚA, 2012, p. $18)^{13}$

Com o banimento e exílio fomos desenraizados, destroncados, eviscerados- fomos arrancados pela raiz, truncados, estripados, despossuídos, e separados de nossa identidade e da nossa história.

12 Tradução livre: A fronteira EUA-México é uma ferida aberta onde o Terceiro Mundo roça contra o primeiro e sangra. E antes de uma sarna formar hemorragias novamente, a força vital de dois mundos se fundindo para formar um terceiro país - uma cultura de fronteira.

${ }^{13}$ Tradução livre: Este livro, então, fala sobre minha existência. Minhas preocupações com a vida interior, e com a luta do eu em meio à adversidade e violação; com a confluência de imagens primordiais; com os posicionamentos originais que a consciência leva a esses fluxos confluentes; e com o meu desejo quase instintivo de se comunicar, de falar, de escrever sobre a vida nas fronteiras, a vida nas sombras. 
Essa citação revela como Anzaldúa expõe seus sentimentos e angústias vividas na fronteira - uma "vida nas sombras", segundo ela mesma. Tais palavras, ditas no prefácio da primeira edição de Borderlands/La Frontera (1987), preparam o leitor para uma narrativa que mistura a vida da autora e sua preocupação com o próximo.

Percebe-se, então, que a literatura de Anzaldúa trabalha nos limites, nas margens, em processo permanente de interação de elementos vários que constituem a identidade e herança cultural do povo mestiço. Dessa forma, apreende-se que o texto de Anzaldúa ganha contornos reais como àqueles de sua vida, sendo parte intrínseca de seu trabalho, mostrando com sua vida e sua auto-história que um mundo sem fronteiras se torna um lugar mais humano.

Com isso, constata-se que o livro Borderlands questionou o paradigma nacionalista cultural que marcava seu local, colocando em foco a produção da mulher latina, lésbica, centroamericana e indígena, o que, por sua vez, nos permite dizer que Anzaldúa também teorizou a intersecção do racismo, sexismo e heterosexismo, ultrapassando as fronteiras estabelecidas na contemporaneidade, dentro de um contexto híbrido e uma consciência opositora ao status quo. Esses conceitos nos possibilitaram enxergar uma consciência de resistência à repressão linguística, cultural e de raça existente, e reconhecer que os entrelugares são formados por essas identidades complexas.

Assim, a cultura do povo mestiço, descrito na obra de Anzaldúa, deixa de ocupar o lugar subalterno para se fazer presente na história dos dois países. A obra Bordelands/La Frontera (2012) representa o direito ao grito de um povo que deseja se descolonizar e ser reconhecido, podendo assim, transitar com o sentimento de estar em seu lugar de direito, não como um forasteiro, ou, nas palavras de Anzaldúa, um "alien element." Trata-se de olhar para o real sentido da problemática que circunda a fronteira: a alteridade. Reconhecer no "outro" mesmo que diferente, um ser que possui a mesma nacionalidade, mas, com identidades diferentes. Para Anzaldúa, "[...] we Chicanos no longer feel that we need to beg entrance, that we need always to make the first overture-to translate to Anglos, Mexicans and Latinos, apology blurting out of our mouths with every step. Today we ask to be met halfway." (ANZALDÚA, 2012, p. 19) ${ }^{14}$

Não obstante, pode-se perceber, nessa passagem, o quanto o discurso de Anzaldúa propõe um posicionamento de respeito entre os povos e suas culturas. Trata-se de ocupar um lugar que não se encontra voltado para um partido de direita ou esquerda, mas sim uma visão diferente, a exemplo do que é postulado pelo estudo descolonial. Há nesse pensamento uma abertura para o outro, para a diferença, ou seja, "la apertura y la liberdad de un pensamiento-outro." (MIGNOLO apud CASTRO-GÓMEZ Y GROSFOGUEL, 2007, p. 31)

\footnotetext{
${ }^{14}$ Esta citação encontra-se no prefácio da $1^{\mathrm{a}}$ edição.
} 
Dessa maneira, a relação entre a intelectual e a nepantlera Gloria Anzaldúa ganha força quando seus textos são aproximados de suas ações enquanto docente e ativista. Em pesquisa aos arquivos da Universidade do Texas, pôde-se ver e ouvir as palestras e aulas de Anzaldúa, momentos nos quais, a intelectual engajada toma forma e consegue, em seu discurso, descolonizar conceitos como o de cultura e identidade, ora tomados como verdade em nossa sociedade contemporânea.

Corroboram com esse pensamento as inferências da Prof. Dr ${ }^{\mathrm{a}}$ Norma Cantú, em entrevista realizada no dia 14 de novembro de 2016, na Universidade Trinity, em San Antonio, Estados Unidos. Segundo Cantú (2016), Anzaldúa era uma "facilitadora"15, seu trabalho realizado na fronteira fez dela conhecedora de todo o contexto existente, conseguindo alcançar a todos com sua linguagem de fronteira e navegar pelos diferentes mundos dos oprimidos. Cantú destaca o fato de Anzaldúa ter vivido na fronteira como peça fundamental nesse processo de produção. Observa também a importância da escrita em Spanglish, ou seja, "esta forma híbrida de falar dos chicanos".

Para Cantú (2016), outra importante contribuição foi a forma com que Anzaldúa conseguia conectar os espaços geopolíticos da fronteira a outros limites, categorias e até a uma visão mundial, global e internacional da forma como nos vemos na Terra. Essa forma de ver e perceber o mundo, segundo Cantú, só foi possível, para Anzaldúa, em virtude de sua espiritualidade.

Segundo a pesquisadora, após mencionar a dificuldade de viver na fronteira dentro de um contexto de discriminação, a exemplo do preconceito contra os homossexuais, afirma que "[...] we had a very hard history in Texas, the repression, the racism, still here, I think it made us stronger, and I know we can survive." (CANTÚ, 2016) ${ }^{16}$ Esse sentimento de luta contra a repressão e racismo na fronteira também são perceptíveis nos textos poéticos da Prof. Cantú, a exemplo do livro Canícula (1995).

Continuando com a entrevista, o Prof. Cantú (2016) corrobora com a reflexão deste artigo ao afirmar que a habilidade de ser uma mestiza, ou seja, conseguir navegar entre diferentes mundos, permitiu a Anzaldúa ser uma ativista (intelectual engajada), não apenas para os chicanos e chicanas, mas para todos aqueles oprimidos, independentemente do nível ou país. Segundo a pesquisadora, "esta é uma razão de Anzaldúa ser popular, pois ela fala para muitos grupos".

Observa-se, por essa perspectiva que o fato de Anzaldúa ter o respaldo de sua herança cultural e social da fronteira e se posicionar como uma mestiza, identifica-a não como uma intelectual marginal, amadora, mas, sim, uma autora dotada de uma linguagem que

\footnotetext{
${ }^{15}$ Facilitadora, neste exemplo, faz referência ao conceito de nepantlera.

16 Tradução livre: [...] nós tivemos uma história muito difícil no Texas, a repressão, o racismo, ainda estão aqui, eu creio que isso nos fez mais fortes, e eu sei que nos podemos sobreviver.
} 
fala a verdade ao poder. Verdade essa reconhecida por tantos grupos até hoje e que tem espaço reconhecido na academia.

Ao ser questionada sobre qual seria a característica mais marcante de Anzaldúa, Cantú (2016) responde que seriam várias, mas destaca o fato de Anzaldúa ser uma mestiça, ser uma facilitadora, trazendo as diferentes visões sobre a fronteira para o debate. Destacou, também, a maneira com que Anzaldúa conectou a temática basilar da fronteira para o espaço geopolítico, como algo maior, "boundaries", e não categorias, expondo de maneira global o que se vive naquele entre-lugar.

Segundo Cantú (2016), "we are a hybrid group", afirmando sobre a mestiçagem de seu povo, que carrega a presença da memória genética tanto de europeus quanto de indígenas. Essa mestiçagem é "parte do que somos" e, por isso, Anzaldúa, como "queer", "tejana", "nepantlera", consegue enfrentar o racismo e navegar por outros mundos e representar todas as pessoas oprimidas.

Contribui para esta reflexão as considerações de Hawley. Para o pesquisador:

\begin{abstract}
Anzaldúa delineates the mutual complicity that negates simple binary splits of White and colored, straight and queer, Christian and Jew, self and other, oppressor and oppressed, since the "insider"/ "outsider" positions are frequently interchangeable. Speaking of a "geography of hybridity", Anzaldúa echoes and informs many postcolonial theorists and critics. She predicts a world, inside and outside the academy, where a "hybrid counsciousness" may be forged. ${ }^{17}$ (HAWLEY, 2001, p. 30)
\end{abstract}

Hawley permite entender que o texto de Anzaldúa não se restringe às categorizações estabelecidas na contemporaneidade, acentuando a necessidade de abertura das barreiras impostas como forma de se (re)pensar o mundo. Trata-se de uma proposta de respeito às diferenças e espaço para uma consciência híbrida.

Nesse sentido, Keating (2009) afirma que se sente golpeada pela forma profunda com que as palavras de Anzaldúa ressoam com tipos de pessoas tão diferentes- não com todos, evidentemente, mas com uma gama surpreendentemente vasta. A pesquisadora observa que os textos de Anzaldúa também incluem muitas pessoas que não se identificam pessoalmente como Chicana, Latina, feminista, lésbica, e/ou gay, eles/elas são tocados pela intimidade e introspecção de Anzaldúa, pois eles/elas sentem como se Anzaldúa

\footnotetext{
${ }^{17}$ Tradução livre: Anzaldúa delineia a cumplicidade mútua que nega simples divisões binárias de brancos e coloridos, retos e estranhos, cristãos e judeus, auto e outros, opressores e oprimidos, uma vez que as posições de "insider" / "outsider" são frequentemente intercambiáveis. Falando de uma "geografia da hibridização", Anzaldúa ecoa e informa muitos teóricos e críticos pós-coloniais. Ela prevê um mundo, dentro e fora da academia, onde um "counsciousness híbrido" pode ser forjado.
} 
falasse diretamente para eles, como se ela estivesse descrevendo seus próprios segredos e pensamentos mais profundos. Keating destaca também que os leitores de Anzaldúa sentem um senso de familiaridade mais intenso do que em outros autores.

Keating (2009) atribui a Anzaldúa a habilidade de gerar tamanha intimidade com os leitores por sua disposição de se arriscar pessoalmente, expor suas verdades, colocar-se "on the line", na linha de frente, empenhando-se para alcançar um grau de "nakedness" 18 , de liberdade de escrita, destituída das máscaras sociais e converções. Conclui Keating, afirmando que: "By plunning so deeply into the depths of her own experiences, no matter how painful those experiences might be, and by exposing herself-raw and bleeding-she externalizes her inner struggles and opens possible connections with her readers. ${ }^{\prime 19}$ (KEATING, 2009, p. 1)

Retomando a entrevista com a Prof ${ }^{a}$. Dr ${ }^{\mathrm{a}}$ Cantú, a pesquisadora afirmou que, ao ler Borderlands/ La Frontera pela primeira vez, reconheceu de que se tratava de algo real, pois naquele momento, estava fazendo parte de uma organização para auxiliar refugiados presos na fronteira, "It was really a hard time to be on the border [...] it was what they were living". Cantú reconhece que o trabalho de Anzaldúa, ao se afastar para conseguir pensar sobre a fronteira foi essencial, pois para ela, estar imersa a todas aquelas necessidades seria impossível pensar em outra coisa, "há muito o que se fazer"[...] "Seria impossível para mim escrever".

Nesse sentido, ao relembrar o papel de Anzaldúa como uma intelectual orgânica que conseguiu representar a sua vida e de seus leitores, Cantú aponta que o engajamento da autora chicana pode ser exemplificado pela forma com que ela conduziu sua vida e projeto intelectual, priorizando àqueles que, como ela, estiveram à margem da sociedade. Ao citar Gramsci e, por extensão, sua perspectiva de intelectual que discute a diferença entre o intelectual que fala para o povo daquele que fala com o povo-intelecutal orgânico, Cantú menciona o quanto Anzaldúa é acessível aos seus leitores. "Ela foi uma de nós."

Entende-se que, ao realizar workshops, palestras e aulas fora do ambiente acadêmico $^{20}$, Anzaldúa conseguiu unir a teoria que desenvolvia na universidade com a prática que a motivava a viver como uma nepantlera. Temas, como, escrita criativa, produção de jornais e poesias, espalharam conhecimento a mulheres, que, a partir dessas aulas, também poderiam ter o seu direito à voz materializado. Contudo, Cantú observa que tais ações não obtiveram reconhecimento da academia; na mesma medida, citou como exemplo, que atualmente o livro de Anzaldúa é lido em universidades que nunca a contratariam, devido à sua forma de ensinar e atuar como uma ativista.

\footnotetext{
18 Tradução livre: liberdade.

19 Tradução livre: Ao penetrar tão profundamente nas profundezas de suas próprias experiências, não importa quão dolorosas aquelas experiências possam ser, e por se expor — cruas e sangrando-ela externaliza suas lutas internas e abre possíveis conexões com seus leitores.

${ }^{20}$ As palestras e aulas eram normalmente realizadas em centros comunitários ou espaços sociais.
} 
Para Malvezzi (2010), ao resgatar as histórias de sua vida e de sua tradição, Anzaldúa, além de toda sua produção, adentrou ao cânone literário para se estabelecer e se firmar como escritora, deixando, assim, a condição marginal a que estava submetida, e, de maneira visível, marcou a apropriação de seu espaço. Para a pesquisadora brasileira, Anzaldúa e Gómez-Peña se apoiam em suas narrativas e no contato com novas condições de cidadania, superando as políticas de desigualdades, por meio de projetos de conciliação e políticas culturais de reconhecimento, ou seja, compartilhando conhecimento com os chicanos e valorizando sua herança cultural.

Observa-se que, ao mostrar novas perspectivas de abordagem da cultura e da tradição dos chicanos, Anzaldúa alcança seu direito ao grito e se materializa como sujeito subalterno que fala a seu povo. Nesse sentido, infere-se, aqui, que o subalterno pode falar e deve lutar para ser ouvido.

Percebe-se assim, que tanto as palavras quanto as ações de Anzaldúa materializam uma leitura de significativa resistência ao poder hegemônico estabelecido, pois, ao possibilitar o (re) pensar das próprias fronteiras de seus leitores é dar novo significado a vida de toda uma comunidade. A exemplo do livro Borderlands/La Frontera, vida e obra de Anzaldúa constituem-se em um legado da história daqueles que estão engajados na fronteira e que percebem a difícil realidade do entre-lugar fronteiriço.

\section{REFERENNCIAS}

ANZALDÚA, Gloria. Borderlands/La Frontera: The new mestiza. $4^{\text {th }}$ edition. San Francisco: Aunt Lute Books, 2012.

BENSON, Library. Texas University at Austin.Gloria Evangelina Anzaldúa Papers, 1942-2004. Disponível em: http://www.lib.utexas.edu/taro/utlac/00189/lac00189.html\#a0 Acesso em: 10-04-2015.

CANTÚ, Norma. Entrevista realizada no dia 14 de novembro na Trinity University, San Antonio, Texas, Estados Unidos, 2016. Duração 52 min.

CASTRO-GÓMEZ, Santiago \& GROSFOGUEL, Ramón. El giro decolonial: reflexiones para una diversidad epistémica más allá del capitalismo global. Bogotá: Siglo de Hombre Editores, Universidad Central, Instituto de Estudios Sociales Contemporáneos, Ponticia Universidad Javeriana e Instituto Pensar, 2007.

HAWLEY, John C. (Editor) Encyclopidia of Postcolonial Studies. Westport, Connecticut, London: Greenwoof Press, 2001. 
KEATING, AnaLouise. The Gloria Anzaldúa reader. Durham and London. Duke University Press, 2009.

MIGNOLO, Walter. El pensamiento decolonial: desprendimiento y apertura. In: CASTRO-GÓMEZ, Santiago \& GROSFOGUEL, Ramón. El giro decolonial: reflexiones para una diversidad epistémica más allá del capitalismo global. Bogotá: Siglo de Hombre Editores, Universidad Central, Instituto de Estudios Sociales Contemporáneos, Ponticia Universidad Javeriana e Instituto Pensar, 2007, p. 25-46.

MALVEZZI, Maria José Terezinha. O eco da voz chicana expressa em singulares (des)caminhos e (des)contextualizações na rede pós-moderna. Tese de doutorado. 367 f. Ibilce-UNESP/São José do Rio Preto, 2010.

NOLASCO, Edgar Cézar. Caldo de cultura: A hora da estrela e a vez de Clarice Lispector. Campo Grande: Editora UFMS, 2007.

SAID, Edward W. Representações do intelectual. Tradução de Milton Hatoum. São Paulo: Companhia das Letras, 2005.

*Professor de Língua Inglesa do IFMS Campus Dourados. Doutorado em Letras pela Universidade Presbiteriana Mackenzie e Mestrado em Letras pela Universidade Federal de Mato Grosso do Sul. Email: carlos.figueiredo@ifms.edu.br

orcid.org/0000-0002-5882-637X

**Professora do Programa de Pós-Graduação em Letras da Universidade Presbiteriana Mackenzie. PósDoutorado pela Brown University-EUA. Doutorado pela Pontifícia Universidade Católica de São Paulo e Mestrado pela Universidade Presbiteriana Mackenzie. Email: verahanna@uol.com.br orcid.org/0000-0002-4395-6702 